\title{
Depth Representation Method by Color Tone for 3D Graphics Modeler
}

\author{
Makoto Fujimura \\ Dept. of Computer and Information Sciences \\ Faculty of Engineering, Nagasaki University \\ Nagasaki, Japan \\ e-mail:makoto@cis.nagasaki-u.ac.jp
}

\begin{abstract}
D computer graphics modeling software that is meant to be operated in a $2 D$ environment comes equipped with a mouse and a 2D display; it is difficult to use these tools to edit 3D mesh objects. This paper proposes an interface for a 3D computer graphics object editing tool to facilitate editing of 3D mesh computer graphics. The interface is able to represent a sense of depth via a perspective view and the gradation of tone color on a 2D display.
\end{abstract}

Keywords-component; $3 D$ CG modeling tool; color tone; a depth perception; monocular stereopsis

\section{INTRODUCTION}

The latest tools available for editing 3D mesh objects have complex interfaces that are capable of constructing orthographic views (front view, top view, side view) and perspective views. 3D computer graphics modeling software that is meant to be operated in a $2 \mathrm{D}$ environment comes equipped with a mouse and a 2D display; it is difficult to use these tools to edit 3D mesh objects. Editing a 3D mesh object is a complex process because it is difficult to locate the depth of a vertex in a $2 \mathrm{D}$ environment. However, the focus of research has not been on editing tools for 3D mesh objects as much as it has been one a geometric modeling[1][2]. Additionally, a vanishing line mesh has been to be effective for depth representation in $3 \mathrm{D}$ scene on a monocular computer display[3].

Depth sensation on a monocular computer display is constructed via certain depth cues[4][5]. These depth cues in human perception are as follows: 1) "Linear Perspective" which implies that parallel lines converge to a vanishing point. 2) "Relative Height and Size," which is represented by changing the location of horizontal line and the object size depending on depth position. 3) The gradient of a color, which serves as a depth cue for humans.

In this paper, we propose a depth control plane in perspective view for virtual $3 \mathrm{D}$ environments, wherein a vanishing line mesh is used as the auxiliary depth coordinate. Further, a depth control plane is used to specify and move the depth position of vertices. Additionally, a sense of depth via color tone is applied to the $3 \mathrm{D}$ computer graphics modeling tool. This proposed depth control plane enables intuitive and efficient interaction in the manipulation of a 3D mesh object.

\author{
Chisa Morishita \\ Dept. of Computer and Information Sciences \\ Faculty of Engineering, Nagasaki University \\ Nagasaki, Japan
}

\section{USERV INTERFACE FOR 3D GRAPHICS MODELING TOOL}

The proposed depth control plan for editing vertices of a 3D computer graphics object, users can perform intuitive manipulation of vertices in one perspective view, which contains a vanishing line mesh and a depth control plane. Figure 1(a) shows an overview of the depth control plane. The vanishing line mesh coordinate explicitly represents the depth of the vertex of the 3D mesh object. The semitransparent yellow rectangle is the depth control plane. In this plane, users can move a vertex arbitrarily by specifying the depth position. Figure 1(b) shows a screenshot of a cube being edited by means of the proposed editing tool. The yellow rectangle of the vanishing line mesh represents the depth control plane. The light yellow polygonal shapes of the cubes represent the depth control plane surfaces of the cubes in cross-sectional planes.

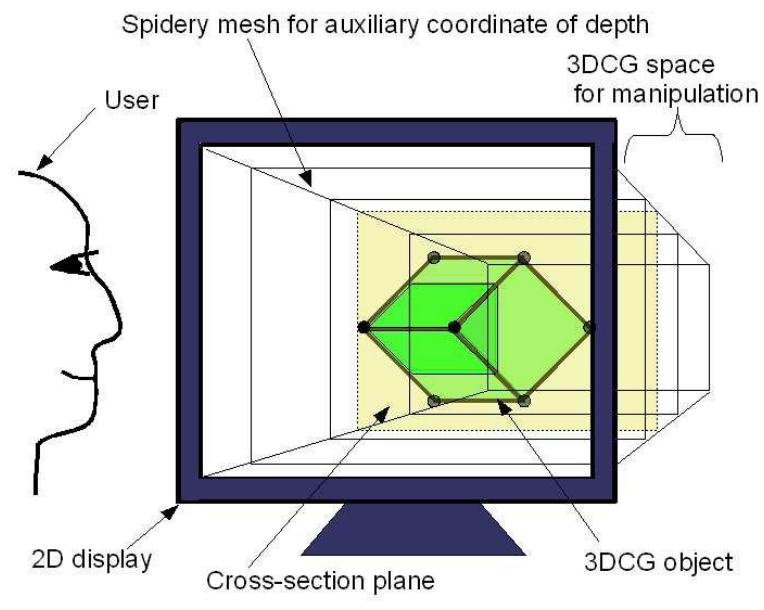

(a)3D graphics modeling tool user-interface by depth control plane 


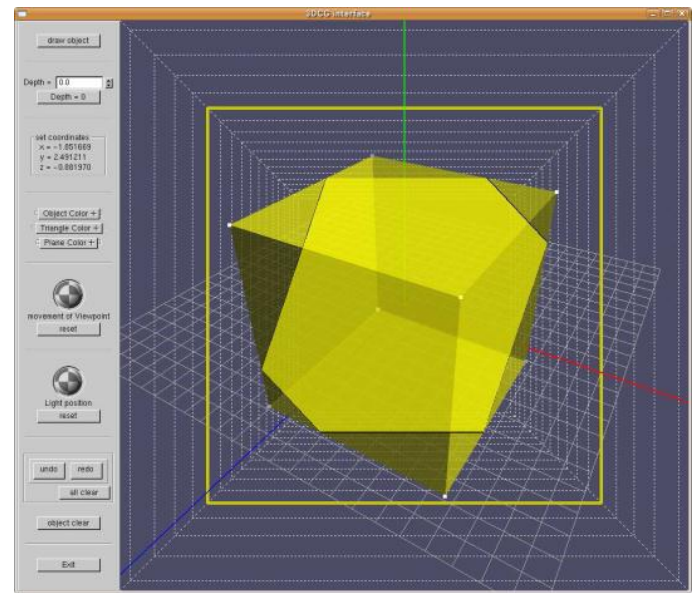

(b)Snapshot of 3D graphics modeling system

Figure 1. Overview of 3D graphics modeling system

\section{DEPTH CONTROL PLANE}

Figure 2 provides an overview of the editing operation for changing the depth of a vertex. Figure 2(a) shows that the vertex is moved from its initial (depth) position to the next (depth) position in the cross-sectional plane by dragging the mouse. The cross-sectional plane is called the "depth control plane.". Next, by moving the cross-sectional plane back and forth, the user can move the vertex in the cross-sectional plane to adjust the depth position. This means that the depth of the vertex can be changed by varying the position of the cross-sectional plane. Figure 2(b) shows how the vertex can be moved in the cross-sectional plane by dragging the mouse. If a user drags the mouse over the vertex on the 2D display, the vertex of the 3DCG object is moved in the depth control plane.

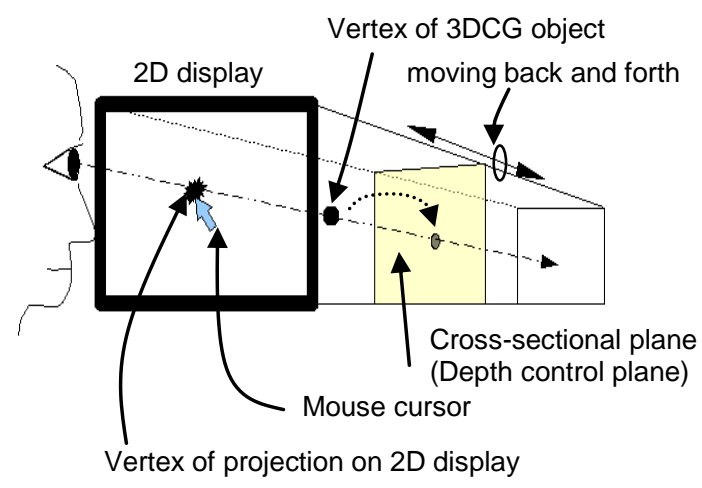

(a)Changing the depth position of the vertex.

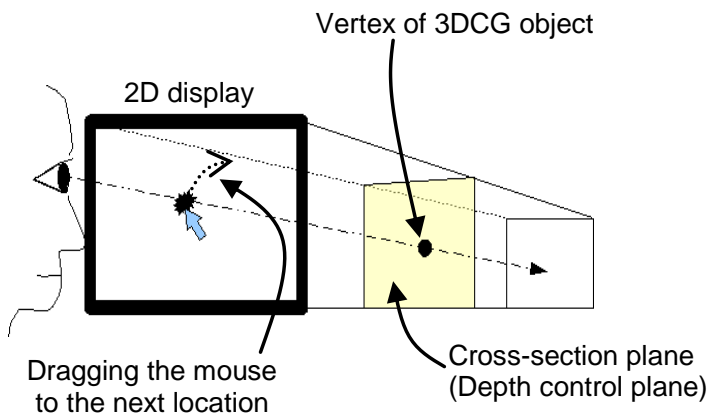

(b)Moving the vertex in the cross-section plane.

Figure 2. Overview of 3D graphics modeling system

\section{DEPTH REPRESENTATION BY COLOR TONE}

\section{A. A sense of depth via color}

Humans are able to perceive a sense of depth through color. One such perception is a sense of depth via gradation of colors, and the other is a sense of depth using warm and cold colors.

Tone gradation is a gradation both in brightness and in saturation. Bright is luminance. Saturation is one of three coordinates in the HSV color space. Warmth color or coldness of color is responsible for the sensation of depth. Warm colors, based on red, orange, and yellow, are called "advancing colors.". Cold colors, based on violet-blue, blue and green-blue, are called "receding colors.".

The depth representation by warmth of color is difficult to control for many 3D computer graphics objects. However, gradation of colors can be controlled easily. Therefore, we use gradation to represent depth.

\section{B. Representation method for a sense of depth}

If there are a number of 3D computer graphics objects to edit, the order of the objects along the depth direction is represented by scaling among the objects, overlapping of the objects, and the degree of transparency. However, these methods of representing a sense of depth have significant effect losses, while the objects are created and edited. Thus, we add the gradation of color effect to the perspective view in the $2 \mathrm{D}$ display.

Figure 3 shows the test images used to evaluate the sense of depth. Figure 3(a) shows "type a," in which three triangles are represented by a different gradation of brightness, depending on their order along the depth dimension. Figure 3(b) shows the same three objects represented by gradation of saturation. Figure 3(c) shows the same three objects represented by gradation of color tone, that is, changes in both brightness and saturation. Figure 3(d) shows the three objects without the use of gradation of color; therefore, they are drawn with the same brightness and saturation. 


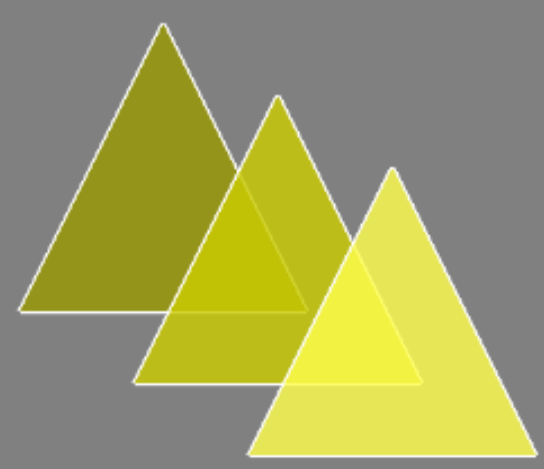

(a) Gradation of brightness

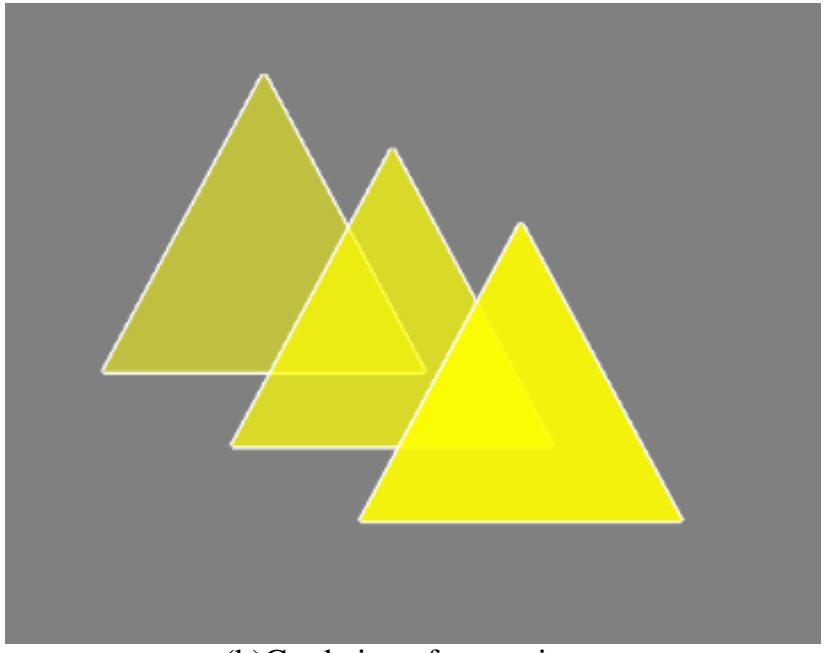

(b)Gradation of saturation

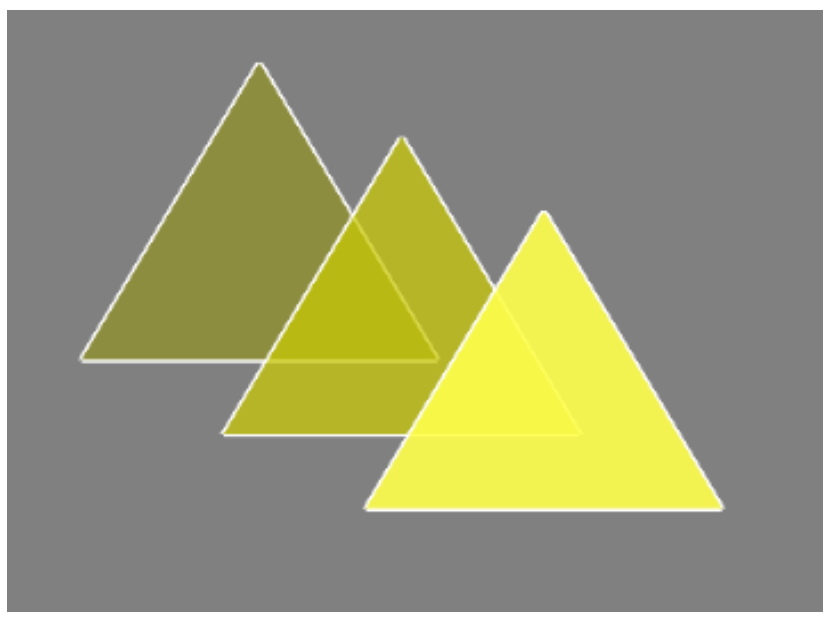

(c)Gradation of color tone

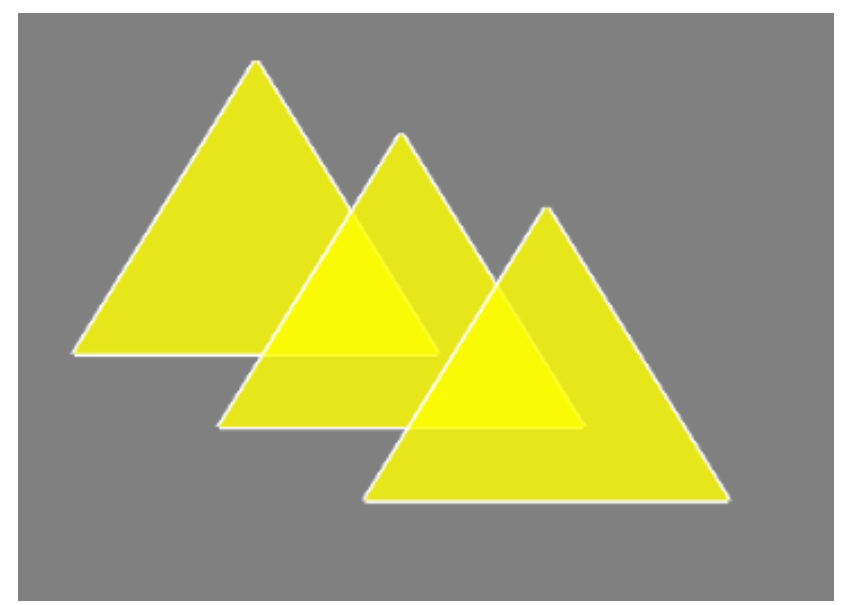

(d)No gradation of brightness, saturation, or color tone

Figure 3. Test images for a sense of depth

\section{Experimental results}

In this experiment, we evaluated the four types of gradation of color shown in Figure 3 so as to adopt the best depth sensation. The test subjects were ten young males. They ranked the four test pictures on a scale of 1 to 4 in the recognition term of order between three triangles.

Figure 4 shows the evaluation results. Type(c) had six first ranks, two seconds ranks and two third ranks. Type (a) had one first rank, eight second ranks, and one third ranks. Type (b) had three first ranks and seven third ranks. Type (d) had ten forth ranks. Thus, type(c) was better adapted to the representation of depth.

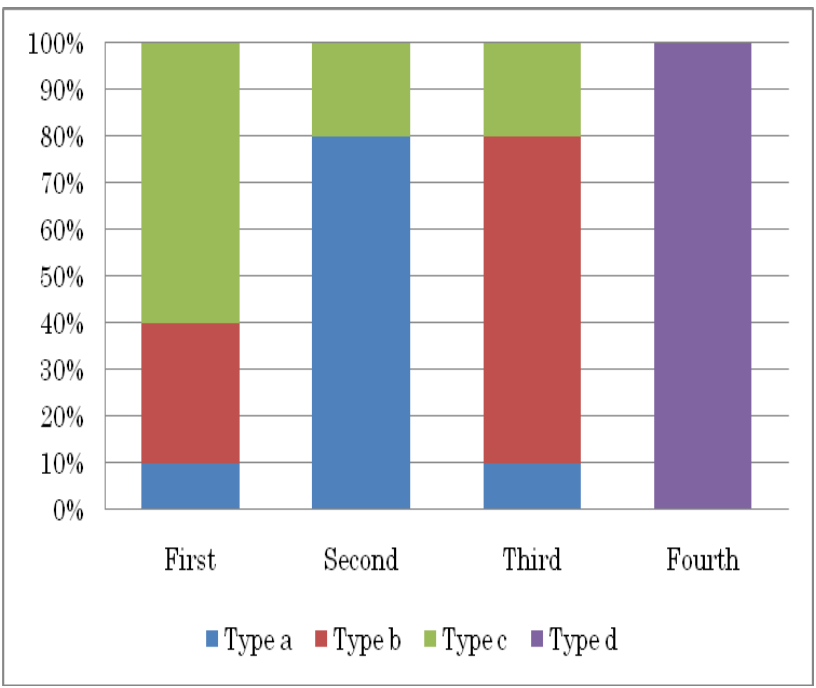

Figure 4. Experimental result 


\section{CONCLUSIONS}

We presented a modeling tool for displaying 3DCG using a cross-sectional plane with a vanishing line mesh coordinate. A depth sensation by gradation of color tone was applied to 3D objects. Our proposed tool provides a more intuitive scheme for the manipulation of the vertices of a 3DCG object. In the future, we will also investigate a more intuitive manipulation method and geometric modeling for vertex manipulation.

\section{REFERENCES}

[1] BENDELS, G. H., AND KLEIN, R.."Mesh forging: editing of 3Dmeshes using implicitly defined occluders.", In SGP '03: Proceedings of the 2003 Eurographics/ACM SIGGRAPH symposium on Geometry processing, Eurographics Association, Aire-la-Ville, Switzerland, Switzerland, pp.207-217, 2003.

[2] ZWICKER, M., PAULY, M., KNOLL, O., AND GROSS, M.,"Pointshop 3D: an interactive system for point-based surface editing.", In SIGGRAPH '02: Proceedings of the 29th annual conference on computer graphics and interactive techniques, ACM, New York, NY, USA, pp.322-329,2002.

[3] HORRY, Y., ANJYO, K.-I., AND ARAI, K. "Tour into the picture: using a spidery mesh interface to make animation from a single image.", In SIGGRAPH '97: Proceedings of the 24th annual conference on computer graphics and interactive techniques, ACM Press/Addison-Wesley Publishing Co., New York, NY, USA, pp.225232,1997

[4] K Oliver, "Depth Perception in Media Design: From Sensory Psychology Cues to Interactive Tools", Journal of Visual Liferacy,Vol.21, No.1, pp.1-14,2001.

[5] Eric C. Banks,"ERNST MACH AND THE EPSODE OF THE MONOCULAR DEPTH SENSATION", Journal of the History of the Behavioral Sciences, Vol.37, No.4, pp.327-348, 2001. 